\title{
Immunology of the ocular surface and contact lens wear: theoretical fundamentals
}

\author{
PIOTR SKOPIŃSKI I, , PIOTR KRAWCZYK ${ }^{2}$ ANNA M. AMBROZIAK ${ }^{2,3}$ \\ ${ }^{1}$ Department of Histology and Embryology, Center for Biostructure Research, Medical University of Warsaw, Poland \\ 2Department of Ophthalmology, Medical University of Warsaw, Poland \\ ${ }^{3}$ Department of Physics, University of Warsaw, Poland
}

\begin{abstract}
Despite constant exposure to the external environment, the ocular surface rarely succumbs to infection. In general, such infection occurs only in the case of mechanical disruption of the epithelial barrier as a result of contact lens wear or injury. Prolonged or overnight contact lens wear resulting in corneal stress may contribute to increased corneal susceptibility to infections and inflammation. Infection can affect both the cornea (infectious keratitis) and conjunctiva (infectious conjunctivitis), but it is the most serious when the cornea is involved because of its role in providing the majority of the refracting power of the eye and in consequence this may lead to loss of visual acuity or even blindness. In this paper we describe a potential effect of prolonged contact lens wear on the pathophysiology of the ocular surface in its immunological and molecular aspects.
\end{abstract}

Key words: contact lens, ocular surface, corneal epithelium, MUC5AC, LTB4.

(Centr Eur J Immunol 2013; 38 (2): 254-259)

\section{Introduction}

It is estimated that 100 million people around the world use contact lenses. This group represents $1.4 \%$ of the total population and only $4 \%$ of 2.4 billion people wearing glasses. Contact lenses, as the optical correction closest to physiology, should therefore be in the near future, the primary means of compensating any defects in vision. In Poland, more than $40 \%$ of the population need vision correction and contact lenses are replacing glasses and becoming a common form of correction of refractive errors.

This paper discusses the basis of the ocular surface immunology with particular emphasis on the specific regulation of the immune response associated with the use of contact lenses.

\section{Ocular surface}

The ocular surface is composed of the corneal epithelium, limbus and conjunctiva (palpebral, forniceal and bulbar conjunctiva). The innate immune system of the eye is similar to other mucosal surfaces. The first tier is passive and consists of several anatomic, physical and chemical barriers that collaborate to prevent infection without inducing inflammation. The second tier is active and consists of cellular and secretory components that together cause acute inflammation aimed at eradicating the pathogen. Despite constant exposure to the external environment, the ocular surface rarely succumbs to infection. In general, such infection occurs only in the case of mechanical disruption of the epithelial barrier as a result of contact lens wear or injury. The defenses confront a considerable challenge and infections become a significant cause of morbidity. Infection can affect both the cornea (infectious keratitis) and conjunctiva (infectious conjunctivitis), but it is the most serious when the cornea is involved because of its role in providing the majority of the refracting power of the eye and in consequence this may lead to loss of visual acuity or even blindness. The tear film coats the epithelia and its complex structure composed of an outer anterior-most lipid component preventing evaporation, an aqueous component with its ions, soluble mucins, enzymes and range of specialized proteins, located directly on the epithelial surface, a thick mucus composed of the gel-forming mucin MUC5AC [1, 2]. The essential function of the ocular surface, epithelia and tears in the 
first place is to create a great barrier that prevents microbial attachment, killing or at least stopping proliferation of constantly attacking organisms, and provide a detection system that in the case of disruption of the primary innate defenses, can activate the adaptive immunity to provide further help to eliminate offending infectious elements.

\section{Tear film}

The tear film is crucial in providing physical defense to the ocular surface. Blinking moves tears into the lacrimal sac, in consequence helping to wash away any potential pathogens before they interact and invade the corneal epithelium. Lacrimal and accessory glands, and ocular surface epithelial cells produce chemical components with antimicrobial properties. Lysozyme was the first indentified enzyme, which was shown to kill Gram-positive bacteria. Secretory phospholipase A2 (sPLA2) has been indentified as the major tear film protein active against Gram-positive bacteria, having no activity against Gram-negative bacteria $[1,3]$.

Other groups of tear film components able to neutralize microbial offenders are the cationic antimicrobial peptides (AMP) and the $\alpha$-defensins human neutrophil peptide (HNP) 1, 2 and 3 (their level rising after the ocular injury).

Another example of cationic proteins found in tears, which is able to interact with bacterial cell membrane causing their lysis, is $\beta$-lysine and secretory leukocyte protease inhibitor (SLP1). Secretory leukocyte protease inhibitor can prevent infection and protect host cells from devastating effects of neutrophil enzymes.

Histatins are small histidine-rich AMPs with antifungal activity detected in tears. Lactoferrin represents over 20 percent of tear proteins and is characterized by high capacity of binding cations; for example iron (essential nutrient), which inhibits bacterial growth. Lactoferrin also has the ability to disrupt the cell membrane of some organisms [1, 4]. The collective family of C-type lectins, surfactant proteins (SP)-A and (SP)-D are other antibacterial molecules contained in the tear film. Surfactant protein A and SP-D are produced by lacrimal gland cells and also corneal and conjunctival epithelial cells. Surfactant protein D is known to inhibit growth of some Gram-negative bacteria, and also to promote pathogen phagocytosis by mononuclear cells. Surfactant protein D inhibits corneal epithelial cell invasion by Pseudomonas aeruginosa probably by way of inhibiting bacterial adhesion to target cells. The predominant immunoglobulin in the tear film is secretory $\operatorname{Ig} \mathrm{A}(\operatorname{sg} \mathrm{A})$. The major effector mechanism of sIgA is neutralization, which prevents attachment to host cells. Secretory IgA is also able to bind lectin-like adhesion molecules on pathogens causing their aggregation and in consequence trapping them within the tear film. Secretory IgA is produced by plasma cells residing in the lacrimal gland and in specialized areas of the conjunctiva referred to as conjunctival-associated lymphoid tissue. Active complement and complement regulatory proteins have also been detected in low levels in tears [5-7].

\section{Epithelial cells}

The superficial layer of epithelial cells is covered with mucus composed majorly of gel forming mucin, MUC5AC, secreted by goblet cells located in the conjunctiva in response to the parasympathetic stimulation. This mucus interacts with the glycocalyx coating the epithelial cells. Mucins are known to help prevent bacteria from reaching the epithelial surface. Mucins have the ability to trap the pathogens, which can be effectively removed from the ocular surface. Secretory IgA, positively charged proteins like lysozyme and SLP1 accumulate in the mucosal layer and provide a variety of antibacterial agents. Bacteria are trapped by the mucins, killed by antibacterial substances, coated with IgA and removed by blinking. Open eye tears have lysozyme, lactoferrin, lipocalin and $\operatorname{sIgA}$ in their composition. Closed-eye tear film has increased amounts of sIgA.

There is a significant migration of neutrophils within 2-3 hours of eye closure, which constitutes a considerable defense factor. It is important to mention the increased levels of such proteins as SLP1 and elafin, which have protease activity, or vitronectin able to inhibit the complement. These proteins help to protect the ocular surface constantly exposed to the proinflammatory environment. Cells composing the superficial epithelial layer of the cornea are bound by tight junctions, sealing them together, creating a mechanical barrier against diffusion of not only fluids, but also microorganisms and their secreted products. Tight junctions also contribute to cell polarity maintenance. Disruption of polarity of these epithelial cells increases susceptibility to infection. Corneal and conjunctival epithelia have a population of stem cells providing new cells to replace those being shed into the tear film $[8,9]$.

\section{Contact lenses}

For over three decades now, an increased risk of corneal infection has been associated with daily and mainly overnight contact lens wear compared with no lens wear. Contact lens has been shown to interfere with most of the physiological functions of the tear film. During eye closure, the physiological environment of the ocular surface undergoes some important changes including hypoxia, reduced $\mathrm{pH}$ and impedance of flushing of debris and proper tear resurfacing of the corneal epithelium [3]. Challenges connected with the contact lens wear are very comparable to those imposed by eye closure. During sleep, neutrophils are thought to take a major role in defending the outer surface of the eye [10-13]. High levels of cytokines and lipid inflammatory mediators are present in tears following eye closure. These mediators are responsible for the recruitment of neutrophils to the ocular surface during eye closure [14-16]. 


\section{Cytokines}

Cytokines produced by a variety of cells play a crucial role in intercellular communication by delivering signals that influence activation, growth, differentiation and migration of target cells. Cytokine signaling between cells often involves a network of effects. At the site of inflammation, recruitment of specific leukocyte populations is connected with the target cell specificity of the individual cytokines or other mediators. In general, overnight contact lens wear modulates the presence of inflammatory mediators and neutrophil numbers. Alteration in the inflammatory cell number may severely reduce the ability of the ocular surface of the clearance of bacteria or debris during sleep [17, 18]. Prolonged or overnight contact lens wear resulting in corneal stress may contribute to increased corneal susceptibility to infections and inflammation. Some studies showed that these two factors; eye closure and hydrogel contact wear promote or modify the growth of the microbiota $[19,20]$. It is well known that after a period of sleep, during extended hydrogel contact lens wear, there occurs the inflammatory disease: contact lens-induced acute red eye (CLARE), manifested by an acute pain, photophobia, reddening of conjunctiva and pronounced lacrimation, with limbal and conjunctival hyperemia, and subepithelial focal and diffuse infiltrates in the cornea. A correlation has been shown between CLARE and Pseudomonas aeruginosa, Serratia marcescens and Haemophilus influenzae, which are able to colonize the contact lens. Another ocular surface trouble associated with improper contact lens wear is CLPU - contact lens-induced peripheral ulcers. Basically it is a self-limiting condition, where corneal changes do not progress beyond a small and peripheral lesion, but may occur because of the full thickness epithelial break in subsequent scarring. The etiology is not fully known, but there are several microbiological demonstrations of contribution of Gram-positive bacteria like Staphylococcus aureus [21, 22].

A large number of studies report the presence of the interleukin 1 (IL-1), IL-6, IL-8 and also granulocyte monocyte colony stimulating factor (GM-CSF) in all layers of human cornea [23, 24]. In vitro studies have shown that addition of exogenous IL-1 or tumor necrosis factor $\alpha$ (TNF- $\alpha$ ) to corneal cell cultures stimulates the synthesis of IL-6 and IL-8 [25]. In response to injury, infection and prolonged contact lens wear, lipid inflammatory mediators may be released by the cornea. Platelet activating factor (PAF) and leukotriene B4 (LTB4) are present on the ocular surface in the case of any inflammation [26]. A strong mechanism of an upregulation of IL-1, IL-6, TNF- $\alpha$, LTB4, thromboxane B2 (TxB2) and prostaglandin E2 (PGE2) has been demonstrated in response to cornel infection of Pseudomonas aeruginosa [27].

Moreover, it has been proved that under specific, stimulated conditions, hypoxia or injury for instance, rabbit epithelial and stromal cells can synthesize PAF and eicosanoids [27]. The effect of these arachidonic acid metabolites on the microvasculature and inflammatory cells overlap proinflammatory cytokines.

In the past, the presence of inflammatory cytokines in tears of individuals experiencing corneal inflammation was reported (TNF- $\alpha$, TNF- $\beta 1$, TNF- $\beta 2$, IL- $1 \alpha$, IL- $1 \beta$, EGF, VEGF, PDGF and HGF) [28-30].

The chemotactic agent associated with CLPU appears to be LTB4, and, to a lesser extent, PAF. Significantly higher levels of both lipid mediators in subjects undergoing CLPU suggest a possible pathophysiological role of LTB4. Leukotriene B4 may be involved in both processes; mainly development and progression of the inflammatory response and infiltration of neutrophils into the corneal stroma [31]. Production of LTB4 may be coupled with PAF synthesis [28]. PAF-like activity in conditions of optimal concentration characteristic of chemotaxis, may be able to induce adhesion molecules on leukocytes and increase vascular permeability and vasodilatation. It is possible that CLPU bacterial products, i.e. toxins, are at the origin of tissue necrosis and may induce production of arachidonic acid metabolites. At the focal site, PMN may be recruited and activated by these proinflammatory mediators. During CLPU, LTB4 and PAF are released by damaged epithelial cells, which then recruit PMN. The stimulus causing the epithelia disruption or damage does not appear to activate PMN or epithelial cells to synthesize cytokines. In an ocular surface inflammation model, LTB4 has been shown to originate from corneal epithelial cells, stromal keratocytes and infiltrating PMN. Increased corneal epithelial production of 12-HETE and 12-HetrE (arachidonic acid metabolites) has been demonstrated in a closed eye contact lens model of corneal inflammation [31-33].

In CLARE, the presence in the tear film of IL-8, LTB4 at submaximal concentrations for chemotaxis and the effect of PAF indicate that these components collaborate to produce an increased chemotactic effect. The crucial stimulus for the synthesis of those mediators seems to be endotoxin/lipopolysaccharide originating from Gram-negative bacteria adherent to the contact lens. Endotoxin is a well-known stimulator of a large number of cells in mammals producing cytokines and arachidonic acid metabolites. The adherent Gram-negative bacteria may release its own chemotactic agents in order to stimulate the infiltrative response. A cytokine playing an important role in CLARE in tears is GM-CSF. This molecule primes PMN for enhanced activity in response to most chemotactic agents [34].

An experimental model in which CLARE tears treated PMN showed an increase in positive cells expressing IgA receptor as compared to untreated PMN and this may indicate that GM-CSF in CLARE tears was functional. GMCSF-primed PMN have been shown to enhance synthesis and release of PAF and LTB4 [35]. Involvement of these mediators does not exclude the probability of other inflammatory mediators playing a role in the initiation of the 
response. A large number of Gram-negative bacteria like Pseudomonas aeruginosa, Serratia marcescens or Haemophilus influenzae adherent to contact lens, may induce production of IL- $1 \beta$ from epithelial cells and may play a major role in early events of the inflammation.

There is a probability that mucosal epithelial cells are activated directly by the bacteria or bacterial products and produce IL- 6 and IL- 8 in the absence of IL- $1 \beta$ or TNF- $\alpha$. Interleukin $1 \beta$ might be downregulated in early stages of inflammatory response [36, 37]. Interleukin 8 and LTB may maintain the PMN response in vivo alone or in combination with other mediators during contact lens-induced inflammatory responses, in CLARE and CLPU.

The presence of IL-8, GM-CSF, LTB4 and PAF-like activity in CLARE tears leads to recruitment and activation of PMN. The lack of corneal disruption or damage during CLARE indicates that the PMN and bacteria are not releasing tissue-damaging enzymes (proteases) or that naturally present inhibitors neutralize their effects.

The presence of increased concentrations of LTB4 and PAF in CLPU tears, and the increased level of LTB4 in comparison with CLARE tears, associated with subsequent stromal scarring may indicate that the PMN activates and releases proteases involved in stromal collagen remodeling, resulting in scarring. In contact lens wearers, PMN seems to be the major defense cell recruited into the corneal stroma. Production of IL-8 and LTB4 may inhibit in a selective manner a PMN response without inhibiting the initiation of the response by other mediators and without impairing the immune response necessary to stop the inflammation.

It is well known that contact lens wear can modify the numbers of PMNs recruited into the cornea during eye closure and also can modulate the concentration of inflammatory mediators in the tear film. During sleep the tear film becomes enriched in $\operatorname{sg} \mathrm{A}$, which can constitute even 80 percent of the total tear protein.

It has also been reported that extended wear contact lens usage may induce Langerhans cells migration into the cornea. Langerhans cells (LC) are specialized, antigen presenting cells that have been histologically localized at a large number of epidermal and mucosal sites, including the eye $[38,39]$. Under normal physiological conditions, those cells, constitutively expressing major histocompatibility class II antigen, are absent from the central cornea [40]. Langerhans cells, which are capable of presenting foreign antigen to CD4+ T cells, are present in the conjunctival epithelium adjacent to the cornea. Various stimuli, like injury, hypoxia or infection cause the cells to migrate from the conjunctiva into the central cornea. Langerhans cells from the conjunctival epithelium are immature with limited antigen presenting functions. Cytokines synthesized in the corneal tissue may have the ability to stimulate migration and maturation of these cells [41]. The density of LC in the central cornea at the time of $\mathrm{T}$ cell infiltration may determine the relative contribution of CD4 and CD8 lymphocytes to the immune response to viral herpetic infection in the cornea.
Extended contact lens wear may constitute a stimulus to induce the Langerhans cells migration into the cornea even after 2-week wear [43, 44]. The LC migration has been reported in another study (guinea pig corneas) after 2-4 days of wear. In users of extended wear contact lenses, the presence of LC in the central cornea can theoretically predispose such eyes to more rapid response to insults.

These cells can initiate antigen processing and enhanced immune responsiveness. In the case of parasite infection (Acanthamoeba keratitis) in the cornea, increased antigen presentation was beneficial in its prevention [44]. But in contrast to the potential benefit of LC in cornea, in immunopathological diseases involving antigen presentation to T cells, LC have been shown to migrate from the eye to the lymph nodes, where they present viral antigen to native CD4+ T lymphocytes which migrate back to the eye, where they enhance further inflammation. In the ocular tissue, the inflammatory component of the immune response may be involved not only in eradication of pathogens, but also can promote damage of host tissue and subsequent impairment of vision. It has been shown that severe, bacterial corneal infection (Pseudomonas aeruginosa) correlates with CD4+ T cells (Th1) regulating inflammatory response. And highly destructive stromal inflammations, potentially leading to perforation are mediated by these cells [45]. The thesis that the extended contact lens wear contributes to the increased risk of LC presentation to T cells in immunedependent and mediated inflammatory responses, may be legitimate [46, 47]. Putting on a contact lens always leads to the disintegration of the tear film, mainly to the dysfunction of its integrity and stability, and in the long-term might also cause the Meibomian gland dysfunction. These conditions are often, apart from nourishing negligence, a starting point of complications associated with contact lens use so you should know the distinct immune surface of the eye and try to act so that it does not interfere significantly and try to avoid damage to its important receptors. Immunology of the ocular surface is an integral part of ophthalmologist's professional knowledge and its understanding should determine a safe, long-term use of contact lenses and also might contribute to the development of new technologies and materials.

The authors declare no conflict of interest. Authors had no financial or proprietary interest in any product, material or method mentioned herein.

\section{References}

1. Dartt DA, Dana R, D'Amore P (2010): Immunology, inflammation and diseases of the eye. Elsevier Ltd.

2. Konat Zorzi G, Contreras-Ruiz L, Párraga JE, et al. (2011): Expression of MUC5AC in ocular surface epithelial cells using cationized gelatin nanoparticles. Mol Pharm 8: 1783-1788. 
3. Song CH, Choi JS, Kim DK, Kim JC (1999): Enhanced secretory group II PLA2 activity in the tears of chronic blepharitis patients. Invest Ophthalmol Vis Sci 40: 2744-2748.

4. Woronkowicz M, Ambroziak AM, Langwińska-Wosko E (2009): Contact lenses and ocular surface pathogens. Kontaktologia i Optyka Okulistyczna 4: 15-19.

5. Ng A, Heynen M, Luensmann D, Jones L (2012): Impact of tear film components on lysozyme deposition to contact lenses. Optom Vis Sci 89: 392-400.

6. Ambroziak AM (2007): Influence of the continuous silicone hydrogel contact lens wear on the corneal physiology with special focus on the tear film evaluation. Kontaktologia i Optyka Okulistyczna 2: 13-19.

7. Phillips TE, Sharp J, Rodgers K, Liu H (2010): M cell-targeted ocular immunization: effect on immunoglobulins in tears, feces, and serum. Invest Ophthalmol Vis Sci 51: 1533-1539.

8. Dartt DA, Dana R (2011): Immunology, inflammation and diseases of the eye. Elsevier Ltd.

9. Ainscough SL, Barnard Z, Upton Z, Harkin DG (2006): Vitronectin supports migratory responses of corneal epithelial cells to substrate bound IGF-I and HGF, and facilitates serumfree cultivation. Exp Eye Res 83: 1505-1514.

10. Dartt DA (2011): Tear lipocalin: structure and function. Ocul Surf 9: 126-138.

11. Lomholt JA, Kilian M (2008): Degradation of uniquely glycosylated secretory immunoglobulin a in tears from patients with Pseudomonas aeruginosa keratitis. Invest Ophthalmol Vis Sci 49: 4939-4944.

12. Redfern RL, Reins RY, McDermott AM (2011): Toll-like receptor activation modulates antimicrobial peptide expression by ocular surface cells. Exp Eye Res 92: 209-220.

13. Jiang X, McClellan SA, Barrett RP (2012): Vasoactive intestinal peptide downregulates proinflammatory TLRs while upregulating anti-inflammatory TLRs in the infected cornea. J Immunol 189: 269-278.

14. Dart JK, Stapleton F, Minassian D (1991): Contact lenses and other risk factors in microbial keratitis. Lancet 338: 650-653.

15. Holden BA, Swarbrick HA, Sweeney DF, et al. (1987): Strategies for minimizing the ocular effects of extended contact lens wear - a statistical analysis. Am J Optom Physiol Opt 64: 781-789.

16. Sweeney DF, Jalbert I, Covey M, et al. (2003): Clinical characterization of corneal infiltrative events observed with soft contact lens wear. Cornea 22: 435-442.

17. Terry RL, Schnider CM, Holden BA, et al. (1993): CCLRU standards for success of daily and extended wear contact lenses. Optom Vis Sci 70: 234-243.

18. Sankaridurg PR, Vuppala N, Sreedharan A, et al. (1996): Gram negative bacteria and contact lens induced acute red eye. Indian J Ophthalmol 44: 29-32.

19. Smolin G, Samy M, Okumoto M, Fuerst D (1984): Effect of soft contact lenses on experimental Pseudomonas and Candida keratitis. Trans Am Ophthalmol Soc 82: 63-74.

20. Turturro MA, Paris PM, Arffa R, Wilcox D (1990): Contact lens complications. Am J Emerg Med 8: 228-233.

21. Cubitt CL, Lausch RN, Oakes JE (1994): Differential regulation of granulocyte-macrophage colony-stimulating factor gene expression in human corneal cells by pro-inflammatory cytokines. J Immunol 153: 232-240.

22. Kernacki KA, Hobden JA, Hazlett LD, et al. (1995): In vivo bacterial protease production during Pseudomonas aeruginosa corneal infection. Invest Ophthalmol Vis Sci 36: 1371-1378.
23. Sheng Y, Birkle DL (1995): Release of platelet activating factor (PAF) and eicosanoids in UVC-irradiated corneal stromal cells. Curr Eye Res 14: 341-347.

24. O'Flaherty JT, Nishihira J (1987): Arachidonate metabolites, platelet-activating factor, and the mobilization of protein kinase $\mathrm{C}$ in human polymorphonuclear neutrophils. J Immunol 138: 1889-1895.

25. Chen SH, Oakes JE, Lausch RN (1993): Synergistic anti-HSV effect of tumor necrosis factor alpha and interferon gamma in human corneal fibroblasts is associated with interferon beta induction. Antiviral Res 22: 15-29.

26. Thakur A, Willcox MD (2000): Contact lens wear alters the production of certain inflammatory mediators in tears. Exp Eye Res 70: 255-259.

27. Kallinikos P, Efron N (2004): On the etiology of keratocyte loss during contact lens wear. Invest Ophthalmol Vis Sci 45: 3011-3020.

28. Schultz GS, Davis JB, Eiferman RA (1998): Growth factors and corneal epithelium. Cornea 7: 96-101.

29. Kallinikos P, Morgan P, Efron N (2006): Assessment of stromal keratocytes and tear film inflammatory mediators during extended wear of contact lenses. Cornea 25: 1-10.

30. Turturro MA, Paris PM, Arffa R, Wilcox D (1990): Contact lens complications. Am J Emerg Med 8: 228-233.

31. Fleischmann J, Golde DW, Weisbart RH, Gasson JC (1986): Granulocyte-macrophage colony-stimulating factor enhances phagocytosis of bacteria by human neutrophils. Blood 68 : 708-711.

32. Agace W, Hedges S, Andersson U, et al. (1993): Selective cytokine production by epithelial cells following exposure to Escherichia coli. Infect Immun 61: 602-609.

33. Weisbart RH, Kwan L, Golde DW, Gasson JC (1987): Human GM-CSF primes neutrophils for enhanced oxidative metabolism in response to the major physiological chemoattractants. Blood 69: 18-21.

34. Weisbart RH, Kacena A, Schuh A, Golde DW (1988): GMCSF induces human neutrophil IgA-mediated phagocytosis by an $\mathrm{IgA} \mathrm{Fc}$ receptor activation mechanism. Nature 332: 647-648.

35. Torres P, de Vos AF, van der Gaag R, Kijlstra A (1994): Expression of the interleukin 1 receptor antagonist in the normal human cornea. Ocul Immunol Inflamm 2: 217-222.

36. Sisson JH, Prescott SM, McIntyre TM, Zimmerman GA (1987): Production of platelet-activating factor by stimulated human polymorphonuclear leukocytes. Correlation of synthesis with release, functional events, and leukotriene B4 metabolism. J Immunol 138: 3918-3926.

37. Nathan H, Naveh N, Meyer E (1994): Levels of prostaglandin $\mathrm{E} 2$ and leukotriene B4 in tears of vernal conjunctivitis patients during a therapeutic trial with indomethacin. Doc Ophthalmol 85: 247-257.

38. Stein RM, Clinch TE, Cohen EJ, et al. (1988): Infected vs sterile corneal infiltrates in contact lens wearers. Am J Ophthalmol 105: 632-636.

39. Williamson JS, DiMarco S, Streilein JW (1987): Immunobiology of Langerhans cells on the ocular surface. I. Langerhans cells within the central cornea interfere with induction of anterior chamber associated immune deviation. Invest Ophthalmol Vis Sci 28: 1527-1532.

40. Hendricks RL, Janowicz M, Tumpey TM (1992): Critical role of corneal Langerhans cells in the CD4- but not CD8-mediated immunopathology in herpes simplex virus-1-infected mouse corneas. J Immunol 148: 2522-2529. 
41. Kelley JG, Ohashi Y, Friedlaender MH (1985): Langerhans cell alterations in the guinea pig cornea. Invest Ophthalmol Vis Sci 26: 1293-1296.

42. Steinman RM (1988): Cytokines amplify the function of accessory cells. Immunol Lett 17: 197-202.

43. Niederkorn JY (2002): Immunology and immunomodulation of corneal transplantation. Int Rev Immunol 21: 173-196.

44. Kwon B, Hazlett LD (1997): Association of CD4+ T celldependent keratitis with genetic susceptibility to Pseudomonas aeruginosa ocular infection. J Immunol 159: 6283-6290.

45. Hazlett LD, McClellan S, Kwon B, Barrett R (2000): Increased severity of Pseudomonas aeruginosa corneal infection in strains of mice designated as Th1 versus Th2 responsive. Invest Ophthalmol Vis Sci 41: 805-810.

46. Wójcik-Gryciuk A, Ambroziak AM (2007): Acanthamoeba keratitis in contact lens users. Kontaktologia i Optyka Okulistyczna 1: 40-42.

47. Chen W, Tang Q, Hendricks RL (1996): Ex vivo model of leukocyte migration into herpes simplex virus-infected mouse corneas. J Leukoc Biol 60: 167-173. 